\title{
La integración de tecnologías digitales en las prácticas de enseñanza de formación inicial docente
}

\section{The integration of digital technologies in the teaching practices of pre-service teacher training}

\author{
Rosana Alvarez \\ Consejo de Formación en Educación - Uruguay \\ rosialv.ros@gmail.com \\ D 0000-0002-5071-4357 \\ Sandra Cabrera \\ Consejo de Formación en Educación - Uruguay \\ sanmacafe@gmail.com \\ iD 0000-0003-3773-1280 \\ Gisella Gonnet \\ Consejo de Formación en Educación - Uruguay \\ profeggonnet@gmail.com \\ iD 0000-0002-5443-6606 \\ Ana Sosa \\ Consejo de Formación en Educación - Uruguay \\ asopri2009@gmail.com \\ 0000-0002-6270-0542 \\ Carina Vázquez \\ Consejo de Formación en Educación - Uruguay \\ cari.ale.vaz@.gmail.com \\ (iD) 0000-0002-1378-1294
}

Fecha de Recepción: 5 de Julio de 2021

Fecha de Aceptación: 6 de Octubre de 2021

\section{Resumen}

Este artículo presenta una investigación que examinó la relación entre la formación inicial docente y la integración de las tecnologías digitales en las prácticas de enseñanza de los estudiantes de cuarto año del Instituto de Formación Docente de Rocha. Se enmarca en el curso Iniciación a la Investigación Cualitativa en Educación y Tecnologías Digitales, coordinado por la Unidad Académica de Tecnología Educativa. Este espacio resultó oportuno para dar lugar a la indagación sobre el grado de integración de tecnologías digitales en las prácticas preprofesionales de los estudiantes 
de cuarto año de Magisterio, interés presente en la comunidad educativa del IFD de Rocha y, particularmente, en quienes integran el equipo de trabajo. La investigación responde a un enfoque cualitativo por medio del estudio de caso. Las técnicas seleccionadas fueron la entrevista en profundidad, la observación, la revisión documental y la encuesta. Como principales hallazgos de esta investigación se identificaron los siguientes factores asociados a la formación inicial docente: la relación teoría-práctica, la confianza y el docente como modelo de enseñanza, todos factores comunes a los tres casos estudiados y que tienen incidencia en la integración de tecnologías digitales. En uno de los casos se identifica además la incidencia de la motivación y en otro la realización de cursos específicos sobre tecnologías digitales.

Palabras claves: Integración de tecnologías digitales, prácticas de enseñanza, formación inicial docente, magisterio.

\section{Abstract}

This article presents a study that examined the relationship between initial teacher training and the integration of digital technologies in the teaching practices of 4th year students of the Teacher Training Institute of Rocha. It is framed in the course Initiation to Qualitative Research in Education and Digital Technologies, coordinated by the Academic Unit of Educational Technology. This space was appropriate to initiate research on the level of integration of digital technologies in the pre-professional practices of 4th year Teacher Training students, a present interest in the educational community of IFD in Rocha and particularly in those who are part of the research team. The research responds to a qualitative approach through a case study. The selected techniques were in-depth interview, observation, documentary review and survey. The main findings of this research identified the following factors associated with Initial Teacher Education: the theory-practice relationship, trust and the teacher as a teaching model, all factors common to the three cases studied and that have an impact on the integration of digital technologies. In one of the cases, the incidence of motivation is also identified, and in another, the realization of specific courses on digital technologies.

Key words: Integration of digital technologies, teaching practices, initial teacher education, teacher training, teaching diploma for primary schools.

\section{Planteamiento del problema}

Esta investigación pretende brindar elementos para la comprensión de las relaciones entre los factores asociados a la formación inicial docente (en adelante FID) y 
los niveles de integración de las tecnologías digitales (en adelante TD) en las prácticas de enseñanza de los estudiantes magisteriales. El punto de partida de este trabajo fue una situación identificada por el cuerpo docente del Instituto de Formación Docente (en adelante IFD) de Rocha. Esta consiste en una manifiesta preocupación por la percepción que tienen los docentes en relación con el bajo nivel de integración de TD en las prácticas de enseñanza de los estudiantes, que asocian, particularmente, a deficiencias en los currículos o en los procesos de enseñanza y de aprendizaje en las asignaturas específicas que la FID brinda: Informática y Educación e Integración de TD. El equipo de investigación se pregunta si estos serían los únicos factores de la FID que influencian el nivel de integración en las prácticas de enseñanza, lo que lleva a un estudio con perspectiva cualitativa que permite profundizar en el fenómeno a fin de comprenderlo.

El contacto con los antecedentes empíricos (Cuadrado, Montaño y Monroy, 2012; Flores-lueg y Roig Vila, 2016; Silva, Rivoir, Onetto, et al, 2017) señala que el bajo nivel de integración en las prácticas de enseñanza es un fenómeno presente en muchas otras realidades institucionales a nivel nacional e internacional, lo que reafirma y hace aún más necesario este ejercicio de comprensión. Esta investigación aporta conocimientos a la comunidad académica, allí donde hoy hay un vacío, enriqueciendo la visión sobre este fenómeno. Por ello, se convierte en un importante insumo para, desde una realidad presente y comprendida, pensar prospectivamente una realidad transformada, lo que aportará a futuras decisiones.

\section{Objetivos de la investigación}

\section{Objetivo general}

Comprender las relaciones entre formación inicial docente y la integración de TD en las prácticas de enseñanza de los estudiantes magisteriales de cuarto grado durante el año 2018 del IFD de Rocha.

\section{Objetivos específicos}

1. Identificar y caracterizar los factores asociados a la FID que promueven la integración de TD en las prácticas de enseñanza de los estudiantes magisteriales.

2. Identificar los niveles de integración de TD en las prácticas de enseñanza de los estudiantes magisteriales. 
3. Analizar las relaciones que existen entre los factores asociados a la FID y el nivel de integración de TD en las prácticas.

\section{Antecedentes y fundamentación teórica}

La creciente expansión de los avances tecnológicos ha modificado la cultura de nuestro tiempo, pues surgen nuevas formas de entender y valorar el mundo en el que vivimos. Autores como Drucker (1969) o Castells (1996) reconocen el desarrollo de una nueva sociedad posindustrial, donde la estructura económica y social se basa en el conocimiento. Castells (1996) sostiene que esta nueva sociedad se basa en un paradigma tecnológico caracterizado por presentarse en dos expresiones: una de producción cultural (a través de Internet y los vínculos sociales que se dan en ella); y otra genética y electrónica mediante la capacidad de recodificar la materia viva, programar, desprogramar y reprogramar. Esta nueva realidad lleva a que se produzcan cambios: mercados globalizados a través de la producción de bienes y servicios; trabajadores cada vez más especializados — pasan de ser las universidades centros de élite a instituciones educativas superiores cada vez más masificadas-; nuevas formas de interaccionar entre las personas, lo que genera nuevas formas culturales; y modificaciones en las políticas económicas, sociales y educativas que intentan atender las problemáticas que surgen ante esta nueva realidad.

En la actualidad, los medios de comunicación digitalizados han relativizado las distancias. Estamos rodeados de herramientas informáticas tales como software, elementos multimedia, computadoras y tabletas portátiles, incluidas ya en el campo educativo, lo que esto hace urgente la efectiva alfabetización digital en la FID.

Un docente alfabetizado logra la integración de texto, sonido e imagen en los documentos multimedia, unido a la interactividad (Gutiérrez, 2000, citado por Del Toro, 2004), porque una vez que se sabe trabajar con las TD en el ámbito educativo, se crean nuevos recursos didácticos, diseñados en forma crítica y responsable, que se integran a la práctica y la vuelven innovadora. Esto requiere repensar los modos y las estrategias que los docentes utilizan para integrar a sus aulas tecnologías educativas digitales. Hargreaves (1998) sostiene que, a pesar de los esfuerzos por formar docentes, los contenidos y prácticas más innovadores se ven suplantados por ideas conservadoras al encontrarse los nuevos docentes en los contextos escolares. 
El campo de la tecnología educativa es muy reciente y, como plantea Litwin (2005), requiere avanzar hacia una construcción enriquecida de su campo de estudio. Dados los avances en las últimas décadas en relación con las nuevas tecnologías, es necesario replantear los modos en que se configuran las prácticas y los roles que en ella se les otorgan. Así, las TD, particularmente vinculadas al contexto educativo, quedan entendidas como el conjunto de herramientas, tanto en el sentido de dispositivos como de aplicaciones, y de líneas teórico-conceptuales sobre su uso, mediadas por la representación de la información en forma binaria, que son ubicadas en un escenario de innovación que potencia los procesos de enseñanza y de aprendizaje.

Al respecto, Gutiérrez (1999) afirma que «la integración de las nuevas tecnologías como medios didácticos, como tecnologías facilitadoras de la enseñanza y del aprendizaje, no es más que un primer nivel de introducción en la educación formal» (p.4). Si este es solo el primer nivel, cuánto más amplio es lo que se pretende como objetivo de la función del educador. La integración de las TD en las instituciones educativas debe pasar por el currículo, por la puesta en marcha de habilidades y destrezas pedagógicas, por la superación de los enfoques tradicionales hacia nuevas metodologías, hacia una perspectiva en la que el docente posea además del conocimiento disciplinar, un solvente conocimiento de la forma en la que se aprende (didáctica y) significativamente, y en las que las TD se tornan invisibles.

Gros $(2000,2004)$ sostiene que la invisibilidad pasa por integrar las TD a la pedagogía y viceversa a través de propuestas en las que estas sean utilizadas para enseñar contenidos curriculares, por lo que dejan de ser un fin en sí mismas y favorecen nuevas formas de enseñanza, para las cuales se requiere un cambio metodológico por parte de los docentes. Adell y Castañeda (2012) y Gros (2015), entre otros, proponen el desarrollo de pedagogías emergentes a partir de nuevos enfoques e ideas en torno a la integración de TD en educación en pos de aprovechar todo su potencial como mindtool o herramienta cognitiva para la creatividad, la colaboración y la productividad multimedia. La integración de TD debe favorecer las relaciones de aprendizaje entre docentes, alumnos y otras redes de aprendizaje a través de un vínculo más transparente que permita el diálogo, la participación y la modificación dinámica de los escenarios de aprendizaje. 
Autores como Area Moreira (2008) y Coll (2008) reconocen que a pesar de que existe un incremento en los recursos tecnológicos en los centros educativos, las prácticas pedagógicas continúan enfocadas en un modelo tradicional. Area Moreira, Hernández Rivero y Sosa Alonso (2016) identifican distintos «usos» en relación con esos recursos tecnológicos. Por ejemplo, con respecto al ordenador, los docentes en sus prácticas de enseñanza lo utilizan como instrumento de investigación por parte de los alumnos (búsqueda de información), como herramienta para la resolución de problemas y la toma de decisiones y como instrumento de producción y recurso de comunicación.

Sánchez llabaca (2003) y Sandholtz, Ringstaff y Dwyer (1997) citados por Parra y Pincheira (s.f) reconocen diferentes niveles para integrar las TIC al aula. En la Tabla 1 se presentan dos de las clasificaciones elegidas como referentes.

Tabla 1

Comparativo de niveles de integración

\begin{tabular}{|c|c|}
\hline Sandholtz, Ringstaff y Dwyer (1997) & Sánchez llabaca (2003) \\
\hline $\begin{array}{l}\text { Entrada: los profesores comienzan a realizar un } \\
\text { uso personal de la tecnología. Inicialmente se } \\
\text { encuentran inseguros, tienen poca confianza en } \\
\text { el uso de los computadores para su trabajo. El } \\
\text { método de enseñanza sigue siendo la } \\
\text { instrucción tradicional, principalmente el dictado } \\
\text { y la clase frontal. }\end{array}$ & $\begin{array}{l}\text { Apresto: iniciación en el uso de las } \\
\text { TIC, en la indagación de las } \\
\text { mismas, sin realizar un uso } \\
\text { educativo; el centro está en las } \\
\text { TIC. }\end{array}$ \\
\hline $\begin{array}{l}\text { Adopción: el computador es usado como } \\
\text { soporte a la clase tradicional; se utilizan } \\
\text { aplicaciones como el procesador de texto. } \\
\text { Existe un alto acceso al computador, pero los } \\
\text { aprendices continúan recibiendo la clase a } \\
\text { través de dictado y métodos tradicionales. }\end{array}$ & $\begin{array}{l}\text { Uso: enfoque tecnocéntrico del uso } \\
\text { de la tecnología para apoyar el } \\
\text { aprender, utilizando las TIC en } \\
\text { distintas tareas, como preparación } \\
\text { de clases, realización de tareas } \\
\text { administrativas, entre otras }\end{array}$ \\
\hline $\begin{array}{l}\text { Adaptación: el computador ha sido integrado a } \\
\text { las clases por los profesores. Su mayor uso se } \\
\text { centra en el procesador de texto, base de } \\
\text { datos, planillas de cálculos y graficadores. La } \\
\text { enseñanza de la sala de clase aún se centra en } \\
\text { la instrucción, el dictado y las formas } \\
\text { tradicionales de enseñanza. Los aprendices }\end{array}$ & \\
\hline
\end{tabular}


Álvarez, Cabrera, Gonnet, Sosa y Vázquez - La integración de tecnologías digitales en las prácticas...

utilizan el computador de manera experimental
como un juego. El computador se utiliza como
apoyo a la actividad de clase, pero se estimula
a los aprendices para que sean creativos en su
uso.

Fuente: elaboración propia a partir de Sandholtz, Ringstaff y Dwyer (1997, citados por Parra y Pincheira, s.f ), y Sánchez Ilabaca (2003).

A los efectos del estudio realizado y luego de analizar los niveles descritos por los diferentes autores presentados en la Tabla 1, este equipo de investigación elaboró cuatro niveles que se presentan en la Tabla 2.

\section{Tabla 2}

Niveles de integración de TD

Niveles

Descripción - Características 
Nivel 1

Rara vez usan las TD, que sirven como complemento de la enseñanza y pueden reforzar habilidades del estudiante, con poca conexión con los contenidos y utilizadas tradicionalmente.

Nivel 2

Usa con cierta frecuencia las TD en su práctica, que sirven para dirigir la enseñanza y como medio de comunicación de los estudiantes. Las TD apoyan la consecución de los objetivos planteados, pero se usan mayormente de forma tradicional.

Nivel 3

Usa las TD cada vez que las necesita con la finalidad de dirigir experiencias con foco en el estudiante que desarrolla habilidades en relación con ellas. Las TD colaboran en la integración de los contenidos y son utilizadas mayormente de forma innovadora.

Nivel 4

Las TD están integradas a la práctica, a consecuencia se desarrollan habilidades en el docente y el estudiante, creando oportunidades de aprendizajes innovadores que no serían posibles sin ellas.

Fuente: elaboración propia.

Este detallado teórico lleva a preguntarse en qué niveles se encuentran las prácticas de enseñanza de los estudiantes magisteriales y cómo influyen en ellas los factores asociados a la FID.

\section{Diseño metodológico: Unidad de análisis, tipo de muestra, instrumentos y validación}

La investigación se desarrolló a través de una metodología cualitativa, la que supone un proceso sistemático conducido con procedimientos rigurosos que permiten la comprensión de las personas, grupos y escenarios dentro de sus marcos de referencia desde una perspectiva holística en el contexto de su pasado y de las situaciones en las que se hallan (Taylor y Bogdan, 2010). Se buscó establecer relaciones entre los factores asociados a la FID y su influencia en la integración de TD en las prácticas de enseñanza, en un proceso comprensivo, desde un diseño emergente que devele vínculos que permitan la comprensión del fenómeno en estudio.

La población está compuesta por cuarenta estudiantes de cuarto año de la Especialidad Magisterio del IFD de Rocha (2018). De esta población, se seleccionó una muestra no probabilística y voluntaria. En forma previa, con la totalidad de los 
estudiantes de cuarto año se realizaron dos instancias de diálogo presentando la investigación e invitándolos a participar de ella. Se presentaron tres voluntarios, por lo cual la unidad de observación (uo) del estudio se conformó por tres estudiantes de cuarto año de Magisterio. La unidad de análisis (UA) es la integración de TD en las prácticas de enseñanza.

El abordaje de la investigación es microsocial, porque busca comprender el funcionamiento dentro de una estructura social determinada, en este caso, del estudiante magisterial en sus prácticas preprofesionales desde un enfoque que se relaciona con las experiencias y perspectiva de los estudiantes y que cobran sentido a partir de su análisis. Las técnicas que dieron respuesta a la pregunta de investigación son: entrevista en profundidad, observación, revisión documental y encuesta. Las entrevistas fueron realizadas a los tres estudiantes (maestros practicantes) que participaron de este estudio de caso y a sus respectivos maestros adscriptores. Por maestro adscriptor se entiende al docente titular del grupo escolar en el cual el maestro practicante realiza su práctica docente durante todo el año. Tiene el rol de enseñar al maestro practicante acerca de cómo abordar contenidos programáticos con los alumnos escolares. Para este instrumento, el equipo de investigación trabajó en duplas. La decisión del trabajo en duplas se tomó en primer lugar por considerar que posibilita pensar y hacer de a dos, brinda seguridad al momento de realizar la tarea, sobre todo tomando en cuenta la falta de experticia del equipo. Esta combinación de dos miradas que focalizan el fenómeno a observar está, por tanto, enriquecida, al dar lugar a puntos de vista que se intercambian mediante la discusión y el debate.

Se efectuaron en campo cuatro observaciones no participantes, realizadas con base en un protocolo previamente elaborado, en el que se consideraron las tecnologías digitales presentes, el desarrollo de la actividad planificada, el rol del maestro practicante y de los alumnos niños, así como también la disposición y la ambientación del salón de clase. Estas observaciones también se realizaron en duplas. Se confrontó la teoría desde la planificación y la práctica desde las prácticas de aula.

Se realizó revisión de dos tipos de documentos: públicos y personales. Fueron estudiados el Plan 2008, en el que se analizó el perfil del egresado, y las planificaciones de los estudiantes magisteriales, que permitieron determinar el nivel de integración. 
Se aplicó una encuesta a directores de las instituciones de práctica (2) y al director del IFD de Rocha para conocer la infraestructura con la que se cuenta en relación a las TD en dichos centros. La encuesta es de tipo autoadministrado y digital (formulario de Google), ya que facilita el acceso y la tabulación de los datos. A partir de ese relevamiento se pudo valorar su incidencia en la integración en las prácticas de aula.

\section{Resultados}

Síntesis de los casos estudiados y discusión de resultados

En los tres casos, los maestros practicantes (en adelante MP) expresan que las asignaturas específicas, Informática y Educación e Integración de TD, aportaron a la integración de las TD en sus prácticas, tanto por la formación desde lo disciplinar, en relación con el contacto con las TD, el conocimiento y dominio como en la aplicación de estas en la práctica. En los tres casos se evidencia que el vínculo teoría y práctica fue un pilar fundamental para planificar actividades con TD, debido a que los estudiantes veían la aplicabilidad del recurso en el aula y eso los motivaba a utilizarlo (por ejemplo, el MP3, referencia que sería de gran importancia trabajar con recursos tecnológicos de manera concreta, desde la didáctica).

La contracara es que el escaso número de actividades con TD observadas por ellos como estudiantes no los motiva a experimentar. Consecuencia de esto es que, si bien se observó que más de la mitad de las planificaciones se sostiene en el uso de TD, el nivel de integración en términos generales es bajo, es decir, se incorporan TD pero no de forma integrada. Otro factor presente en los tres casos es el de docentes como modelos que favorecen u obstaculizan la integración de TD. El docente constituye un modelo dado que es una función inherente al vínculo alumno-docente, de la que no se puede escapar; aunque la maestra adscriptora (en adelante MA) diga «no sirvo como modelo» (entrevista MA1), lo es. También cabe señalar la manifiesta necesidad de los MP de tener modelos a seguir, porque este factor queda intrínsecamente relacionado con la confianza.

Respecto a los docentes del IFD los MP afirman que solo integran videos y presentaciones estilo PowerPoint en sus prácticas, con un uso básico y tradicional de las TD. Los tres entrevistados manifiestan como excepción la asignatura Lenguas Extranjeras, donde en coordinación con el equipo рот, trabajaron durante todo el año 
en la plataforma CREA2, por lo cual lograron ver la aplicabilidad de las TD en el aula. Se destaca que el MP2 reconoce en primer lugar a este docente como modelo. También se hace una alusión específica a Matemáticas de segundo por parte del MP3.

En este punto, es significativo vincular las expresiones de los MP sobre la escasa integración de las TD y su uso básico con el equipamiento con el que el IFD cuenta. EI relevamiento muestra un importantísimo equipamiento: cada aula cuenta con televisor que incluye cables de conexión para los diferentes puertos (atendiendo a los diversos modelos de laptop), hay conexión a internet de media a buena calidad en todos los espacios, salón específico para uso de TD muy bien equipado, sala de videoconferencias (vc), cuatro cañones, una pizarra digital, kits de robótica y de sensores fisicoquímicos. Se cuenta, además, con cuatro docentes que integran el equipo DOT.

Por otro lado, se debe hablar del modelo desde los MA. Es notoria la escasez de experiencias con integración de TD en aula que recuerdan los MP. Se suma a esta escasez la propia negación del MP1 como modelo, el uso «básico y tradicional» que los MP relatan y el hecho de que aun cuando se les sugiere por parte del MA el uso de determinado recurso, ellos no lo vieron aplicado. En las prácticas que se identifican con TD, el recurso más utilizado por los MP para sus planificaciones con TD son los videos. Nos parece destacable que tanto el MP2 como el MP3 se arriesgan a utilizar recursos más allá de lo que modélicamente han percibido, aunque el MP3 tenga en su experiencia las devoluciones del MA del año anterior, las que considera significativas ya que en la entrevista refiere que le indicaban si el recurso utilizado era correcto o no.

Además del vínculo teoría-práctica y la figura del docente como modelo, la confianza es otro factor que incide en la integración de TD por parte de los MP. La confianza se presenta como un estado interpersonal, que afecta el sentido que los sujetos le otorgan a lo que hacen brindando una sensación de seguridad. Al integrar TD, la confianza se observa relacionada con dos aspectos. Por un lado, asociada con el dominio instrumental de los recursos tecnológicos. Desde este aspecto la confianza incide en la toma de decisiones a la hora de integrar o no recursos tecnológicos en sus prácticas. Los entrevistados afirman que necesitan dominar las TD para poder llevarlas al aula. Cuando no hay dominio se trabaja con ellas mecánicamente, como se observó en las actividades áulicas del MP1 y MP2. En ellas se desarrolla un libreto con las TD del que no pueden salirse, pues no tienen la confianza para trabajar con los emergentes de 
la clase; impiden que se realicen tareas fuera del tiempo que van marcando, por tanto, los alumnos no tienen espacio para salirse de lo propuesto, incluso se les indica volver para atrás. El MP3, al respecto, sostiene que prefiere no exponerse a integrar herramientas que no domina, una idea similar se evidencia en el MP2, quien afirma que recién en su cuarto año se «animó» a usar las tabletas portátiles.

Por otro lado, la confianza no solo está vinculada al dominio de las herramientas, sino también a la relación teoría-práctica. Los MP afirman que en las asignaturas específicas alcanzaron conocimiento y dominio de algunos recursos que se potencian al ser visualizados en la práctica, como manifiesta el MP3: al verlos «aterrizados» adquieren sentido. Reconocen que esa formación no es suficiente y que, en realidad, no puede pretenderse que dos años permitan adquirir experticia, más aún si se considera que el ámbito de las TD se encuentra en constante cambio y evolución. El MP2, refiriéndose a los dos años de formación en asignaturas específicas, expresa:

Para mí está bien, pero me gustaría... [...] los cuatro años me parece que serían fundamentales, porque en realidad tenemos Informática, pero más acerca de la tecnología, de las plataformas y de todo eso lo tuvimos el años pasado y fue un año solo (entrevista MP2).

Tanto en el MP2 y MP3 se observa capacidad reflexiva a la hora de articular los contenidos disciplinares con los saberes sobre el uso de la tecnología, potenciando así los procesos de aprendizaje. Incluso en el MP2 se visualiza una crítica respecto a la selección y al uso de videos poco adecuados para el logro de los objetivos planteados.

En relación con los criterios para valorar la adecuación, selección y uso de las TD, los MP no tuvieron devolución de sus MA.

Resulta interesante que, a diferencia del MP1, que usa las TD para que los alumnos estén ordenados ${ }^{1}$, el MP2 considera que los alumnos se motivan mucho más con el video que con otro recurso. Una situación similar se evidencia en el MP3 quien afirma que el docente debe «tratar de atrapar a la mayor cantidad posible» de niños (entrevista MP3).

\footnotetext{
${ }^{1}$ Uso de TD orientado a controlar la conducta de los alumnos según el MP1.
} 
La Figura 1 muestra el número de planificaciones con TD (62) en el total de las planificaciones analizadas (105). Es significativa la presencia de TD en los tres casos, en más de la mitad del total de planificaciones.

\section{Figura 1}

Total de planificaciones y total de planificaciones con integración de TD por caso.

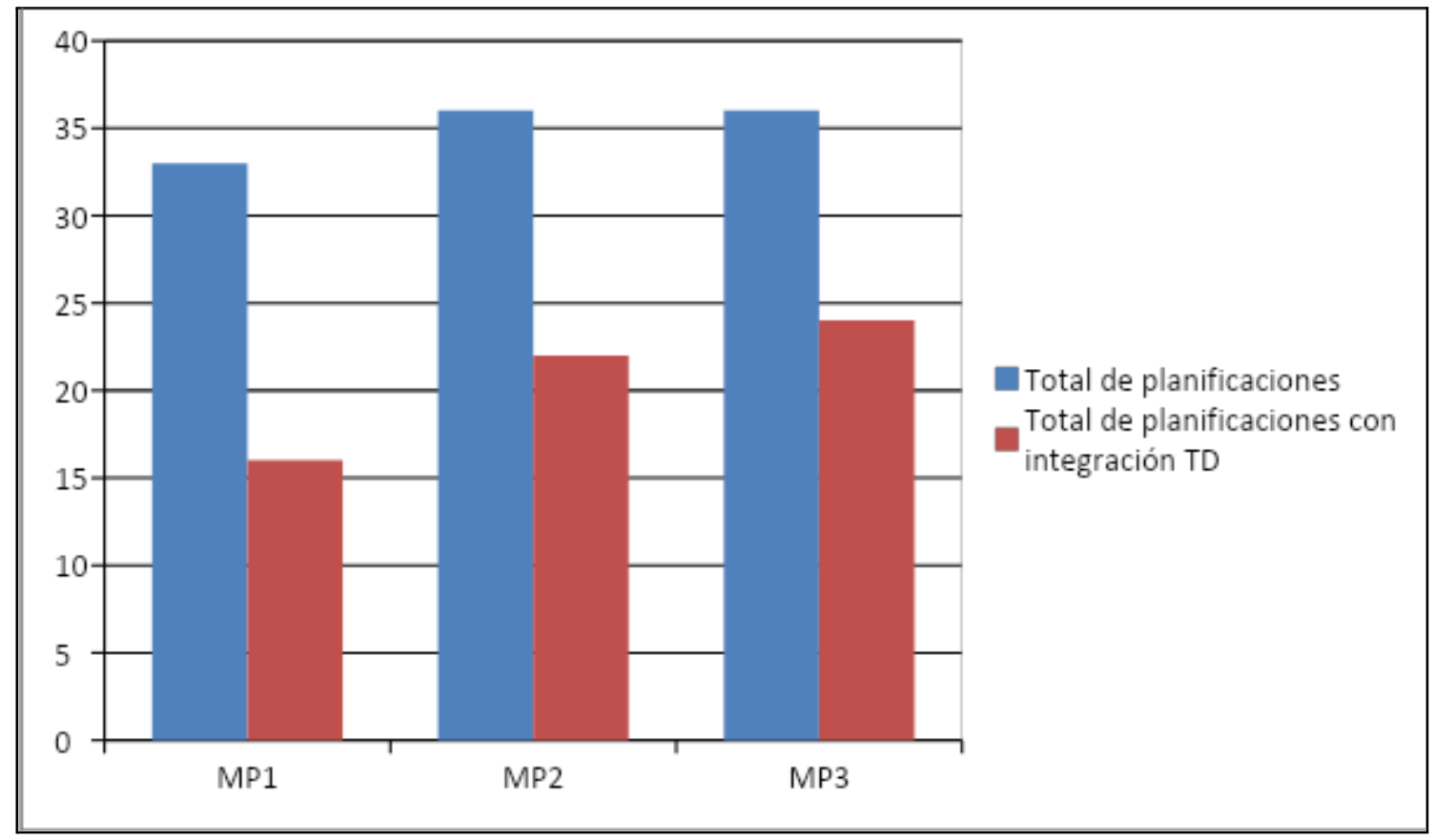

Fuente: elaboración propia.

Al relacionar el número de planificaciones con TD y el área para la cual se planifica, se observa que en la que más se integra es en Conocimiento de la Naturaleza. En menor grado, se trabaja en las restantes áreas, siendo la menos contemplada el Área de Ciencias Sociales. Es significativo señalar que en los casos de los MP1 y MP2, se hace una integración más abarcativa de todas las áreas de conocimiento, mientras que el MP3 se centra solo en tres de ellas (Figura 2). 
Figura 2

Número de planificaciones con TD según área de conocimiento, por caso.

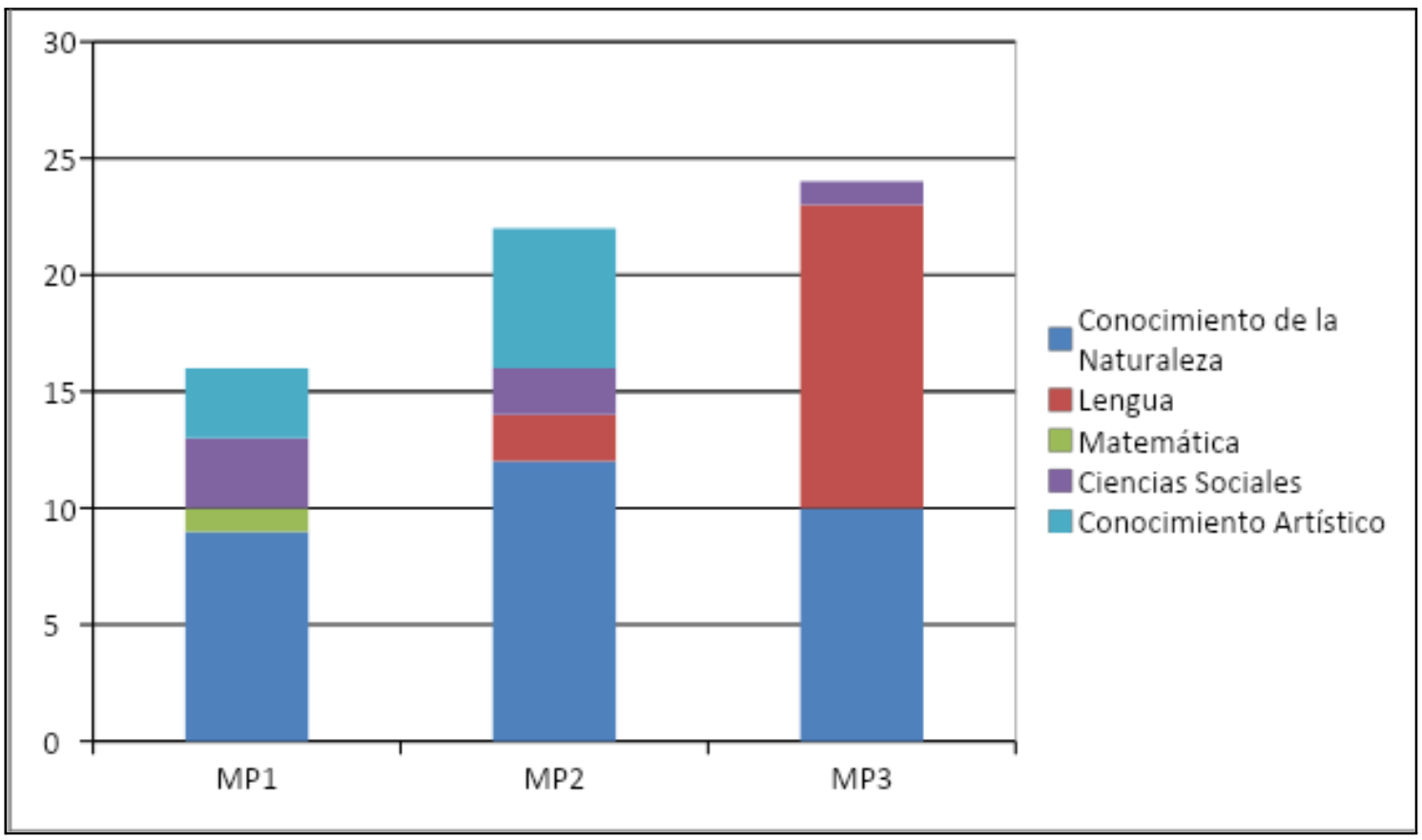

Fuente: elaboración propia.

Refiriéndonos a la integración de las TD que realizan los MP, se identifica el uso del video como un aspecto que se reitera en los tres casos y se utiliza principalmente para abordar Conocimiento de la Naturaleza; particularmente en el caso del MP3, solo se lo integra en esa área. Coincidentemente, los tres MP lo usan para confrontar hipótesis, dos de ellos además como disparador (MP1 y MP3), mientras que el MP2 lo emplea también como cierre, con la función de institucionalizar, como fuente de información. A pesar de la coincidencia en el área del conocimiento y en el propósito con el que se integra, el manejo del video es realizado por el MP3 de manera diferente.

Al hablar del uso o propósito con el que las TD se integran al aula, se llega al rol desempeñado por el alumno niño en relación a ellas. Resulta interesante observar que del total de planificaciones con TD (62), la mayoría son con video (44), en las que el alumno niño no desempeña un rol de gestión o interacción con las TD, es mero espectador. 
Si se relacionan las planificaciones con TD y el tipo de recurso utilizado en ellas, resulta claro que el video es el recurso estrella. Aparecen con menor presencia las tabletas y el hipertexto (Figura 3).

Figura 3

Número de planificaciones según recurso utilizado por caso.

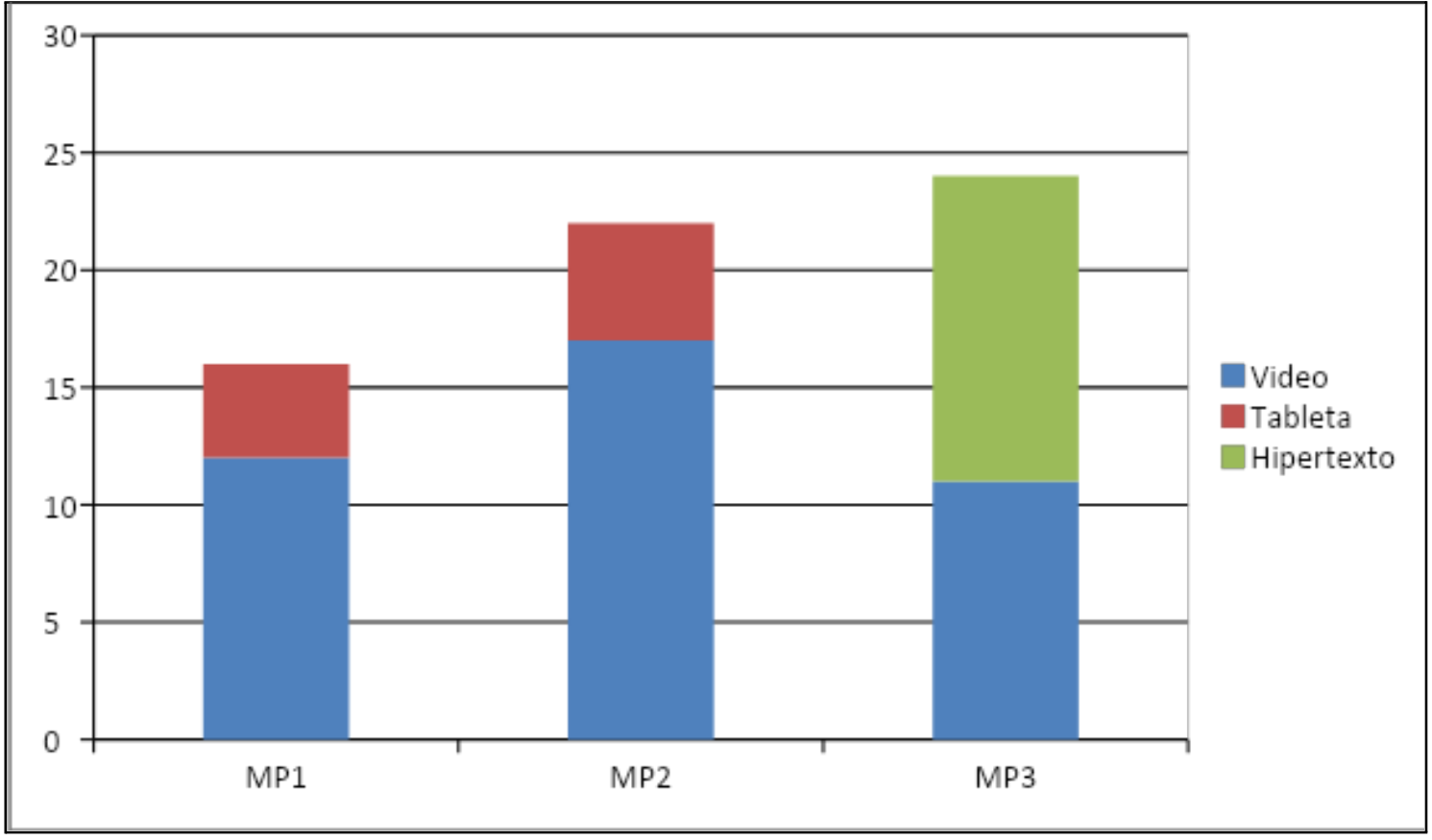

Fuente: elaboración propia.

Una vez triangulados los datos recogidos de las entrevistas a los MP y a los MA, de las observaciones de clase y las planificaciones de los MP, con los factores asociados a la integración de las TD, se relacionaron los niveles de integración identificados por este equipo con el perfil de egreso del Plan Integrado de Formación Docente, 2008.

El perfil de egreso (general a todas las carreras) establecido en dicho Plan, no contempla la necesidad de que el egresado desarrolle habilidades y competencias digitales. Si el perfil de egreso que constituye la referencia y guía del plan de estudios (en el que el ítem específico de perfil de egreso magisterial está sin desarrollar) no tiene en cuenta explícitamente la formación con TD, difícilmente los docentes de las diferentes asignaturas, en especial las no específicas, pondrán foco en dicha formación. 
Al triangular los tres casos en los niveles de integración (página 12) alcanzados en sus prácticas y relacionados con los factores identificados, es importante destacar que en los casos 1 y 2 alcanzan el nivel 2 e incluso en el MP2 se presentan elementos del nivel 3. Mientras que en el caso 3, en el cual la motivación es señalada por el propio estudiante como factor decisivo, es en el que se detecta un mayor nivel de integración, quien alcanza un nivel 3. Incluso explora, llevado por su propia motivación, e integra en sus planificaciones TD que no había observado en sus modelos.

En los tres casos estudiados, se constata que los docentes de asignaturas no específicas, salvo en Lenguas Extranjeras y Matemática, no integran las TD o lo hacen esporádicamente empleando videos y presentaciones. Es en las asignaturas específicas, Informática y Educación e Integración de TD, de segundo y de tercer año respectivamente, en las que manifiestan haber recibido diversos niveles de formación en el abordaje con TD.

Según el análisis realizado de los objetivos de los programas de dichas asignaturas, en Informática se consideran las TD como herramientas de apoyo para la preparación de clase y contenidos, lo que coincide con la percepción que tienen los MP en cuanto a su aplicabilidad en sus prácticas de enseñanza. Con respecto al objetivo general del programa de Educación e Integración de TD, se desprende que la finalidad es la formación pedagógica y tecnológica para promover el desarrollo de una comunidad docente que dé respuesta a las exigencias de nuevos entornos.

Si bien existen diferencias en cuanto a los recursos tecnológicos que poseen las diferentes instituciones en las que los MP realizan Su FID, en los tres casos solo usan dos TD, el video y la tableta (MP1 y MP2), y el video y el hipertexto (MP3).

\section{Conclusiones}

Esta investigación se inició con el objetivo de establecer relaciones entre la FID y la integración de las TD en las prácticas magisteriales e identificar los factores asociados a la FID que inciden en la integración de las TD, comprendiendo esas relaciones y reconociendo niveles de integración. En relación con la formación que reciben como estudiantes durante cuatro años, es importante señalar que el contacto con asignaturas específicas en TD es en segundo año con Informática y en tercer año con Educación e Integración de TD. Los MP consideran que dos años no son suficientes y que sería fundamental tener formación durante los cuatro años de la carrera. No obstante, también señalan que no debe esperarse que la FID los convierta en expertos, en primer 
lugar porque la actitud que el MP toma frente a las TD influye y es personal y, además, porque el propio escenario de las TD está en permanente evolución. Resulta interesante que los MP manifiesten que recibieron formación en las asignaturas específicas, pero no puedan detallar qué es para ellos "aprendí muchísimo», o que no recuerden el nombre de las aplicaciones que consideran adecuadas para integrar a sus prácticas. No existe una correspondencia entre lo manifestado en las entrevistas, las observaciones de clases y el análisis de las planificaciones, ya que solo utilizan dos recursos digitales y con un uso mayormente tradicional.

Fuera de la formación recibida en las asignaturas específicas, la integración de TD en el aula es percibida por los MP en Su FID como muy escasa, que se integra con usos básicos y tradicionales, al punto de calificar esas clases como monótonas. Estos resultados coinciden con la realidad presentada en los resultados de uno de nuestros antecedentes (Frutos, 2014). Solo dos cursos (el de Lenguas Extranjeras y el de Matemática) son percibidos por ellos como espacios donde se desarrollan prácticas innovadoras con TD. Si bien los MP manifiestan críticamente que el uso de las TD que realizan sus docentes de asignaturas no específicas es básico, tanto en el IFD como en la escuela, el uso que ellos ponen en práctica al momento de enseñar tiende a ser similar a dicho modelo.

Tres son los factores que se identificaron presentes en los tres casos: el docente como modelo de enseñanza, la relación teoría-práctica y la confianza. En uno de los casos se identificó, además, como factor asociado, la motivación y, en otro caso, la realización de cursos extracurriculares específicos.

Al relacionar el nivel de integración de cada MP con los factores asociados a su FID, se pone de manifiesto que en los tres casos el modelo de enseñanza es relevante, tanto en el «ejemplo a seguir» (MP1 y MP2), como así también en la necesidad manifiesta del MP3, quien expresa necesitarlo y no tenerlo. Se evidencia que el vínculo teoría-práctica es fundamental como soporte para la integración de TD. LoS MP manifiestan que cuando ven la TD vinculada a la práctica le encuentran sentido. A su vez, si en la práctica ven actividades de enseñanza exitosas sin uso de TD tienden a replicarlas, quedándose en la zona de confort. Difícilmente integrarán TD en sus prácticas preprofesionales si la mayoría de los modelos que tienen plantean actividades sin uso de TD. Por lo tanto, se considera que la relación entre teoría y práctica es un 
factor asociado a la FID que incide en la integración de TD por parte de los MP en sus prácticas.

La relación entre la confianza, el docente como modelo y el vínculo entre teoría-práctica es estrecha además de interdependiente. Tales elementos no contribuyen a la integración de TD, tal como se analizó en los tres casos. Para que los MP integren las TD en su aula, la formación, además de ser sistemática, debe apuntar a la relación teoría-práctica. No es suficiente tener más asignaturas específicas, sino que toda la formación dialogue con modelos para los MP.

En uno de los tres casos analizados se presenta la motivación como un factor determinante para la integración de TD. Esta motivación está dada desde dos aspectos: en el primero, desde el punto de vista del alumno niño, dado que las TD ingresan al aula para motivar a los alumnos en la realización de las propuestas. El MP3 manifiesta que en las prácticas en las cuales usa TD (donde el juego digital es una de ellas), aquellos niños que normalmente no son tan partícipes en el aula se presentan aquí interesados, participativos y expectantes por la actividad a realizar. El MP2 considera que con el video aparece una motivación mayor que con el uso de otros recursos. En el otro aspecto, la motivación del MP aparece cuando establece que, si el colectivo al que pertenece promueve e incentiva el uso de TD, favorece que otros las usen, se motiva por tanto a crear y utilizar recursos digitales para el abordaje de contenidos.

Los factores mencionados en los párrafos anteriores inciden en el nivel de integración de las TD por los MP, que se encuentran entre los niveles 1 y 3 de la categorización realizada por este equipo de investigación. En dicha categorización, se tiene en cuenta la frecuencia de uso de la TD, el propósito, la forma en la que se integran y la relación de los contenidos programáticos.

En cuanto a la frecuencia de integración de TD, en dos de los tres casos se utilizan con cierta frecuencia, siendo el video el recurso más utilizado, aproximadamente en las tres cuartas partes de las planificaciones analizadas, en las que se destaca, además, que el video no es de elaboración propia. El video se integra con el propósito de mantener el orden y la concentración, así como para abordar contenidos programáticos. En este último punto se integra con las siguientes finalidades: como disparador, fuente de información y también como institucionalización de los contenidos. Este recurso se emplea usualmente para abordar contenidos del Área del 
Conocimiento de la Naturaleza, que corresponde a más de las tres cuartas partes de las planificaciones con TD.

El uso de la tableta portátil, utilizada por dos de los tres casos, se remite a la búsqueda de información, producción de texto y elaboración de tablas para abordar contenidos en el Área del Conocimiento de la Lengua. En solo uno de los casos se emplea la página web para abordar contenidos de Lengua desde la tipología textual digital.

La forma en la que se integran las TD en las secuencias de actividades mayoritariamente se observa como inmutable y rígida. Se constata la misma secuencia de actividades en casi toda la documentación analizada, especialmente para el video. El uso de las TD no resulta innovador en la mayoría de los casos, aunque corresponde destacar que en el MP3 se observa cierto grado de innovación por la forma en la que usa el video para su análisis y el uso de la página web para desarrollar competencias digitales en sus estudiantes, aspectos por los que se le asignó el nivel 3 de integración de las TD. Ninguno de los casos estudiados alcanza el nivel 4, ya que no se observan prácticas con TD que generen oportunidades de aprendizajes innovadoras. En la mayoría de las prácticas observadas y analizadas, las actividades presentadas con integración de TD podrían haber sido realizadas prescindiendo de ellas, por lo que no cumplen su rol. Reafirma esta idea el uso que se observa en dos de los casos estudiados, donde las TD ingresan en el Área del Conocimiento Artístico en sentido reproductivo y no creativo, en tareas que naturalmente deberían potenciar la creatividad.

Al analizar el equipamiento en TD con el que cuentan las escuelas en la que se realiza la práctica de cuarto año magisterial y el IFD, se desprende que no existe una relación directa entre cantidad y variedad de equipamiento con el nivel de integración de las TD. Es de destacar la riqueza de equipamiento en TD que tiene el IFD, que está a disposición de los practicantes y docentes de otras instituciones. Por lo expuesto, tanto la integración de la TD de los docentes del IFD y de los MA como de los MP, no está limitada por la falta de equipamiento.

Sucede algo similar con los dispositivos Ceibal, ya que el obstáculo no es la posesión de la herramienta, sino que los alumnos no traen la laptop a clase, viene descargada, sin cargador o rota. Este hecho constituye otro tipo de barrera, en tanto se 
cuenta con los dispositivos, pero las situaciones que se detallan son obstáculos que están bajo la supervisión de las familias. Cuando se habla de brecha, se entiende en un primer momento la falta de acceso a la herramienta, pero en estos casos la herramienta está, por lo que la brecha se da en su uso no integrado o directamente la escasa frecuencia con la que se usa. Como conclusión, se necesita no solo el acceso a las TD, sino su uso frecuente e integrado que desarrolle habilidades en el docente y el estudiante y genere aprendizajes innovadores que no serían posibles sin ella.

La presente investigación permitió, a través del estudio de tres casos, comprender las relaciones que existen entre la FID y la integración de TD en las prácticas de enseñanza de los estudiantes magisteriales de cuarto año del IFD de Rocha, donde los modelos de enseñanza constituyen, sin lugar a dudas, el factor de mayor incidencia a la hora de integrar las TD, factor que está íntimamente ligado a la relación teoría-práctica.

\section{Limitaciones del estudio}

Se destaca entre las limitaciones del estudio que la fecha de realización del trabajo de campo coincidió con el cierre del año lectivo de los MP (demora producida por la espera de los permisos correspondientes para ingresar a las instituciones de práctica), por lo tanto, no hubo tiempo para realizar un mayor número de observaciones. La participación de los MP que formaron la muestra fue voluntaria, por lo que se contó con menos individuos a investigar de los que este grupo esperaba. Se pensaba en una muestra de ocho a diez sujetos de la población total (40) y solo se obtuvieron tres voluntarios. Otra limitación del estudio tiene que ver con que el ma1 es compartido en dos de los MP.

\section{Referencias bibliográficas}

Adell, J. y Castañeda, L. (2012). Tecnologías emergentes, ¿pedagogías emergentes? En J. Hernández, M. Pennesi, D. Sobrino y A. Vázquez (Coord.). Tendencias emergentes en educación con TIC. Barcelona: Asociación Espiral, Educación y Tecnología. pp. 13-32.

Area Moreira, M (2008). La innovación pedagógica con TIC y el desarrollo de las competencias informacionales y digitales. Investigación en la escuela, (64), pp. 5-17

Area Moreira, M, Hernández Rivero, V., Sosa Alonso, J. (2016). Modelos de integración didáctica de las TIC en el aula. doi: https://doi.org/10.3916/C47-2016-08 
Castells, M (1996), La era de la información: Economía, Sociedad y Cultura. (Vol. 1). Alianza Editorial.

Coll, C. (2008). Aprender y enseñar con las TIC. Expectativas, realidad y potencialidades. Boletín de la Institución Libre de Enseñanza, (72), pp. 17-40.

Cuadrado, I., Montaño, A. y Monroy, A. (2012). Las TIC en la Formación Inicial del Profesorado. ICTs In Teacher's Initial Training. En: Morales, J; Barroso, J. (2012). Redes Educativas: la educación en la sociedad del conocimiento. Sevilla GID.

https://idus.us.es/xmlui/bitstream/handle/11441/56729/OPO\%2011.\%20Las\%20TIC\%20 en\%20la\%20formaci\%C3\%B3n\%20inicial\%20del\%20profesorado.pdf?sequence=1\&i sAllowed=y

Del Toro, M. (2004). Una propuesta dirigida a la formación de la competencia para el diseño didáctico del contenido digital de cursos de postgrado asistidos por multimedia interactivo. [tesis doctoral, Instituto Superior Politécnico José Antonio Echeverría].

Drucker, P (1969). The Age of Discontinuity. Harper \& Row.

Flores-lueg, C. y Roig Vila, R. (2016). Percepción de estudiantes de Pedagogía sobre el desarrollo de su competencia digital a lo largo de su proceso formativo. Estudios pedagógicos, 42(3), 129-148. http://dx.doi.org/10.4067/S0718-07052016000400007

Frutos, L. (2014). La inclusión del uso didáctico de las TIC en Formación Docente Inicial para Enseñanza Media. Oportunidades y desafíos en la construcción del oficio de enseñar. [Tesis para obtener el título de Máster en Educación. Universidad ORT].

Gros, B. (Coord.). (2000). El ordenador invisible, hacia la apropiación del ordenador en la enseñanza. Barcelona: Ed. Gedisa.

Gros, B. (2004) De cómo la tecnología no logra integrarse en la escuela a menos que... cambie la escuela. https://cdn.educ.ar/repositorio/Download/file?file_id=5728a709-5aa5-4403-83ca-d28 $2 \mathrm{~d} 2 \mathrm{c} 7 \mathrm{dd} 9 \mathrm{e}$

Gros, B. (2015). La caída de los muros del conocimiento en la sociedad digital y las pedagogías emergentes. Education In The Knowledge Society (EKS), 16(1), 58-68. doi:10.14201/eks20151615868

Gutiérrez, A. (1999). Educación y Nuevas Tecnologías. La Obra. Revista de Educación, 75 (898).

Gutiérrez, A. (2000). Comunicación multimedia, interactividad y aprendizaje. (tesis doctoral). Universidad de Valladolid. 
Hargreaves, A. (1998) Dimensiones subjetivas e institucionales de la formación docente, en Birgin, A., Dussel, I., Duschatzky, S. y Tiramonti, G., La formación docente. Bs.As.: Troquel.

Litwin, E. (Comp.) (2005) Tecnologías educativas en tiempos de Internet. Bs.As.: Amorrortu editores.

Parra, E. y Pincheira, R. (s.f). Integración curricular de las TIC. Universidad Católica de la Santísima

Concepción.

https://www.oas.org/cotep/GetAttach.aspx?lang=es\&cld=412\&aid=707

Sánchez Ilabaca, J. (2003). Integración curricular de TIC. Concepto y Modelos. Enfoques Institucionales, 5(1).

https://enfoqueseducacionales.uchile.cl/index.php/REE/article/view/47512/49550

Silva, J., Rivoir, A., Onetto, A., Morales, M. y Miranda, P. (2017). Estudio comparado de las competencias digitales para aprender y enseñar en docentes en formación de Uruguay y Chile. Fondo Sectorial de Educación. Inclusión Digital Educación con Nuevos

Horizontes.

https://digital.fundacionceibal.edu.uy/jspui/bitstream/123456789/219/1/FSED_2_2015 _1_109518.pdf

Taylor, S. J., Bogdan, R. (1984). Introducción a los métodos cualitativos de investigación. México: Paidós. 